\title{
AN EXAMPLE ON NULL SETS OF PARABOLIC MEASURES
}

\author{
JANG-MEI WU
}

(Communicated by J. Marshall Ash)

\begin{abstract}
A set $E$ on the real line of Hausdorff dimension 1 is constructed, such that the graph of $E$ on the boundary of any $C^{2}$ domain $\{t>\tau(x)\}$, or on the boundary of any Lip $\frac{1}{2}$ domain $\{x>\chi(t)\}$, is null with respect to the parabolic measure associated with any parabolic operator $L=a(x, t) \partial^{2} / \partial x^{2}-$ $\partial / \partial t$ on $\mathbf{R}^{2} ; a$ is Hölder continuous and $0<\Lambda_{1} \leq a \leq \Lambda_{2}<\infty$ for some constants $\Lambda_{1}$ and $\Lambda_{2}$.
\end{abstract}

This note concerns parabolic operators $L$ on $\mathbf{R}^{2}$ of the form

$$
L=a(x, t) \frac{\partial^{2}}{\partial x^{2}}-\frac{\partial}{\partial t},
$$

where $a$ is Hölder continuous and satisfies

$$
0<\Lambda_{1} \leq a(x, t) \leq \Lambda_{2}<\infty
$$

for some constants $\Lambda_{1}$ and $\Lambda_{2}$.

For such operators, the maximum principle on bounded domains and the Harnack principle are classical. The Dirichlet problem is solvable on rectangular domains-the base is regular because $a(x, t) \leq \Lambda_{2}$ and the sides are regular because $a(x, t) \geq \Lambda_{1}$.

In a domain $\Omega$ defined by $\{t>\tau(x)\}$ with $\tau$ continuous on $-\infty<x<\infty$, or defined by $\{x \leq \chi(t)\}$ with $\chi \in \operatorname{Lip} \frac{1}{2}$ on $-\infty<t<\infty$, the maximum principle is still valid; and the Dirichlet problem, with continuous, compactly supported boundary data, is uniquely solvable (to be verified in $\S 6$ ). Therefore, the value of a solution at a point $(x, t)$ can be treated as a linear functional on the space of the boundary values, and can be represented by a probability measure $\omega_{\Omega}^{(x, t)}$ on the boundary. It is called the parabolic measure associated with $L$; and for a Borel set $E \subseteq \partial \Omega, \omega_{\Omega}^{(x, t)}(E)$ is a solution of $L=0$ on $\Omega$.

In [1], Fabes and Kenig proved that on the domain $\{t>0\}$, there exists a parabolic operator $L$, with coefficient $a$ uniformly continuous in $\{t>0\}$, so that the associated parabolic measure is purely singular with respect to the Lebesgue measure on $\{t=0\}$.

Received by the editors October 4, 1988.

1980 Mathematics Subject Classification (1985 Revision). Primary 35K10, 31C99.

The author was partially supported by the National Science Foundation. 
It is proved in [4] that on the domain $\{t>0\}$, for any $\delta>0$, there exists a parabolic operator $L$, such that the corresponding parabolic measure is concentrated on a set of dimension $<\delta$. It is not clear whether a similar result holds on $\{x>0\}$. However, there exist a $\operatorname{Lip} \frac{1}{2}$ domain $\{x>\chi(t)\}$ and a set $E$ on the boundary, of dimension $<1$, so that all the parabolic measures associated with the heat equation vanish off $E$ ([3]).

On half planes $\{t>-M x\}$ or $\{x>0\}$, all boundary sets of zero $\Lambda_{1} / \Lambda_{2}-$ dimensional Hausdorff measure have zero parabolic measure associated with any parabolic operator satisfying (0.2) - a consequence of Lemma $\mathrm{D}$ below and the maximum principle.

In this note, we construct a set $E$ on $\mathbf{R}$ of Hausdorff dimension 1, such that the graph of $E$ on the boundary of any $C^{2}$ domain $\{t>\tau(x)\}$ (or of any $\operatorname{Lip} \frac{1}{2}$ domain $\left.\{x>\chi(t)\}\right)$ is null with respect to the parabolic measure associated with any parabolic operator $L$. More precisely, we prove

Theorem 1. Given any Hausdorff measure function $h(s)$ with $h(s) / s \rightarrow+\infty$ as $s \rightarrow 0^{+}$, there exists a compact set $E$ on $\mathbf{R}$ of infinite Hausdorff measure $\tau(x) C^{2}$ Lambda $_{h}(E)$, such that on any domain $\{t>\tau(x)\}$, with $t=\tau(x) C^{2}$ on $-\infty<x<\infty$, (respectively, $\{x>\chi(t)\}$, with $x=\chi(t)$ Lip $\frac{1}{2}$ on $-\infty<$ $t<\infty)$, the parabolic measure associated with any parabolic operator $L$ vanishes on $\{(x, \tau(x)): x \in E\}$ (respectively, $\{(\chi(t), t): t \in E\})$.

On $C^{2}$ domains $\{t>\tau(x)\}$, or domains $\{x>\chi(t)\}$ slightly better than Lip $\frac{1}{2}$, the parabolic measure associated with the heat equation is known to be mutually absolutely continuous with respect to the Lebesgue measure of the projection of the boundary set onto $t=0$, or $x=0$ respectively ([5], [6]).

In $\S 2-5$, we give the proof of Theorem 1 ; in $\S 6$, we substantiate the existence and the uniqueness of the Dirichlet problem, which have some independent interest. Propositions 2 and 3 are about parabolic measures on $C^{2}$ and $\operatorname{Lip} \frac{1}{2}$ domains, and are workable substitutes for the doubling property.

The author is grateful to the referee for the following remark: when the domain is $\{t>\tau(x)\}$ or $\{x>0\}$, the theorem is valid if $a(x, t)$ is only uniformly continuous-parabolic measures exist under these circumstances. In fact, smooth compactly supported functions on the boundary have extensions to $\mathbf{R}^{2}$ which lie in the Sobolev space of functions with one time derivative and two space derivatives in $L^{2}$. Based on a lemma of Stroock and Varadhan [1, Lemma 0], the solution of the Dirichlet problem exists in this Sobolev space. The maximum principle follows by smoothing the coefficients and taking the limit of the solutions.

\section{THE SET $E$}

Let $h$ be a Hausdorff function with $h(s) / s \rightarrow \infty$ as $s \rightarrow 0^{+}$. Replacing $h(s)$ by $\inf _{0<i \leq 1} h(t s) / t$ if necessary, we may assume that $h(s) / s$ is decreasing. We choose a continuous increasing function $f$ on $[0,1]$ with $f(0)=0, f\left(\frac{1}{2}\right)=1$ 
and $h(s f(s)) / s \rightarrow \infty$ as $s \rightarrow 0^{+}$; and integers $j(n), n=1,2,3, \ldots$, so that $j(1)=1$ and $f\left(2^{-j(n)}\right) / f\left(2^{-j(n-1)}\right) \leq 1 / n$. We define $\alpha_{j}=\frac{1}{2}$ for $j \neq j(n), n=$ $1,2,3 \ldots$, and choose numbers $\alpha_{j(n)}$ inductively so that

$$
\prod_{1}^{j(n)} \alpha_{j}=2^{-j(n)} f\left(2^{-j(n))}\right), n=1,2, \ldots
$$

Note that $\alpha_{1}=\frac{1}{2}$,

and

$$
\alpha_{j(n)} \leq \frac{1}{2 n}, \quad n=1,2, \ldots,
$$

$$
h\left(\prod_{1}^{j(n)} \alpha_{j}\right) 2^{j(n)} \rightarrow \infty \quad \text { as } n \rightarrow \infty .
$$

Let $\mathscr{F}_{0}=[0,1]$. And let $\mathscr{F}_{k}(k \geq 1)$ consist of $2^{k}$ closed intervals in $[0,1]$ with mutually disjoint interiors, of length $\prod_{1}^{k} \alpha_{j}$ each, constructed inductively as follows: for each interval $I$ in $F_{k-1}$, we subdivide $I$ into two equal intervals if $\alpha_{k}=\frac{1}{2}$; subdivide the middle $2 \alpha_{k}$ portion of $I$ into two equal intervals if $\alpha_{k}<\frac{1}{2}$. These new intervals constitute $\mathscr{F}_{k}$. Let

$$
E=\bigcap_{1}^{\infty} \mathscr{F}_{k} \text {. }
$$

It is clear from (1.1) that the Hausdorff measure $\Lambda_{h}(E)=\infty$.

Denote by $\Delta(x, r)=\{y:|y-x|<r\}$ and we have

Proposition 1. Let $E$ be the set constructed above and $\mu$ be a measure on $[0,1]$. Suppose that there exist $K>1, \varepsilon_{0}>0$, a closed set $Z$ on [0,1], and a function $\alpha$ which is positive continuous on $[0,1] \backslash Z$, and equals one on $Z$, such that for any $0<\varepsilon<\varepsilon_{0}, 0 \leq x \leq 1$, and $0<r \varepsilon^{-K}<R \leq \alpha(x)$,

$$
\mu(\Delta(x, r)) \leq C \varepsilon \mu(\Delta(x, R)),
$$

where $C$ is a constant independent of $x, \varepsilon, r, R$, and $\alpha$. Then $\mu(E)=0$.

Proof. Given $0<\varepsilon<1$, let $N=\left[\varepsilon^{-K-10}\right]$, where [ ] is the greatest integer function. We cover $E$ by a collection $\mathscr{I}$ of intervals from $\cup_{n \geq N} \mathscr{F}_{j(n)-1}$ with mutually disjoint interiors, so that if $I \in \mathscr{I} \cap \mathscr{F}_{j(n)-1}$ and $J_{I}^{1}$ and $J_{I}^{2}$ are the two intervals in $\mathscr{F}_{j(n)}$ that are contained in $I$, then either (a) $\left(J_{I}^{1} \cup J_{I}^{2}\right) \cap Z \neq \emptyset$, or (b) $\left(J_{I}^{1} \cup J_{I}^{2}\right) \cap Z=\emptyset$ and $|I| \leq \inf _{I} \alpha(x)$. This is possible because $\alpha(x)$ is positive continuous on $[0,1] / Z$.

Let $\mathscr{J}=\left\{J_{I}^{k}: I \in \mathscr{J}, k=1,2\right\}$. Clearly, $\mathscr{J}$ is a covering of $E$ and $\left|J_{I}^{k}\right| /|I| \leq 1 / N<\varepsilon^{K+9}$ for any $I \in \mathscr{I}$. Therefore from (1.2) we obtain

$$
\mu\left(J_{I}^{k}\right) \leq C \varepsilon \mu(I) \quad \text { for } I \in \mathscr{I}, k=1,2 .
$$

Thus $\mu(E) \leq C \varepsilon$. Hence $\mu(E)=0$. 


\section{Proof of TheOREM 1}

It is apparent that we only need to prove that the parabolic measures in question have the properties of $\mu$ in Proposition 1. Fix a parabolic operator $L$ on $\mathbf{R}^{2}$ satisfying $(0.2)$.

Let $t=\tau(x)$ be a $C^{2}$ function on $\mathbf{R}, \Omega=\{t>\tau(x)\}$ and $\omega$ be the parabolic measure associated with $L$ on $\partial \Omega$. Because parabolic measure zero is a local property, we assume, as we may, that $\sup \left|\tau^{\prime \prime}\right|<+\infty$; let $A=1+\sup \left|\tau^{\prime \prime}\right|$ and

$$
\alpha(x)= \begin{cases}1, & \tau^{\prime}(x)=0, \\ \min \left\{1, \frac{\left|\tau^{\prime}(x)\right|}{400 A^{2}\left(\Lambda_{1}+1\right)}\right\}, & \tau^{\prime}(x) \neq 0 .\end{cases}
$$

We denote by $\Phi(x, r)=\{(y, \tau(y)):|y-x|<r\}$ and by capital $C$ positive constants depending at most on $\Lambda_{1}, \Lambda_{2}$, and $A$. We have

Proposition 2. Under the above assumptions, let $0<\varepsilon<10^{-5}, x_{0} \in \mathbf{R}, 0<r<$ $R<\alpha\left(x_{0}\right)$, and

$$
\frac{r}{R}<\frac{\Lambda_{1}}{\Lambda_{2}} \varepsilon^{2+\Lambda_{2} / \Lambda_{1}} .
$$

Then

$$
\omega^{(x, t)}\left(\Phi\left(x_{0}, r\right)\right) \leq C \varepsilon \omega^{(x, t)}\left(\Phi\left(x_{0}, R\right)\right)
$$

for $(x, t) \in \Omega \backslash \Gamma$, where

$$
\begin{aligned}
\Gamma & =\left\{(x, t):\left|x-x_{0}\right|<\varepsilon R,\left|t-\tau\left(x_{0}\right)\right|<\left(A+\frac{1}{\Lambda_{1}}\right) R^{2}\right\}, \quad \tau^{\prime}\left(x_{0}\right)=0 ; \\
& =\left\{(x, t):\left|x-x_{0}\right|<\sqrt{\Lambda_{1}\left|\tau^{\prime}\left(x_{0}\right)\right| R / 4,}\right. \\
& \left.\left|t-\tau\left(x_{0}\right)\right|<\left|\tau\left(x_{0}-\frac{\Lambda_{1} R}{10^{4} \Lambda_{2} \log \frac{1}{\varepsilon}}\right)-\tau\left(x_{0}\right)\right|\right\}, \quad \tau^{\prime}\left(x_{0}\right) \neq 0 .
\end{aligned}
$$

In particular, for any $x_{0}$ in a closed interval $\left[x_{1}, x_{2}\right],(2.2)$ holds for every $(x, t) \in \Omega$ with

$$
t>\tau\left(x_{1}\right)+2\left(1 / \Lambda_{1}+\left|\tau^{\prime}\left(x_{1}\right)\right|+A\right)\left(1+\left|x_{2}-x_{1}\right|^{2}\right) .
$$

Fix a $\operatorname{Lip} \frac{1}{2}$ function $x=\chi(t)$ on $\mathbf{R}$ with

$$
|\chi(t)-\chi(s)| \leq B|t-s|^{1 / 2}, \quad t, s \in \mathbf{R},
$$

for some $B>0$, denote by $\widetilde{\Omega}$ the domain $\{x>\chi(t)\}$, and let $\widetilde{\omega}$ be the parabolic measure associated with $L$ on $\partial \widetilde{\Omega}$. Denote by $\Psi(t, r)=\{(\chi(s), s)$ : $|s-t|<r\}$ and by lowercase $c$ positive constants depending at most on $\Lambda_{1}, \Lambda_{2}$, and $B$. We have 
Proposition 3. Under the above assumptions on the Lip $\frac{1}{2}$ domain $\{x>\chi(t)\}$, let $0<\varepsilon<B^{2} / 4000\left(\Lambda_{2}+B^{2}\right), x_{0} \in \mathbf{R}, 0<r<R<1$ and

$$
r / R<\varepsilon^{2+2 \Lambda_{2} / \Lambda_{1}} .
$$

Then

$$
\widetilde{\omega}^{(x, t)}\left(\Psi\left(t_{0}, r\right)\right) \leq c \varepsilon \widetilde{\omega}^{(x, t)}\left(\Psi\left(t_{0}, R\right)\right)
$$

for $(x, t) \in \widetilde{\Omega} \backslash \widetilde{\Gamma}$, where

$$
\widetilde{\Gamma}=\left\{(x, t):\left|x-\chi\left(t_{0}\right)\right|<B \sqrt{R} / 2 \text { and }-r<t-t_{0}<\frac{B^{2} R}{4000\left(\Lambda_{2} \log \frac{1}{\varepsilon}+B^{2}\right)}\right\} \text {. }
$$

In particular, for $t_{0}$ in a closed interval $\left[t_{1}, t_{2}\right],(2.4)$ holds for all $(x, t) \in \widetilde{\Omega}$ with $t>1+t_{2}$.

It follows immediately from Propositions 1,2 , and 3 and the Harnack principle that $\omega(\{(x, \tau(x)): x \in E\}) \equiv 0$ on $\Omega$, and that $\widetilde{\omega}(\{(x(t), t): t \in E\}) \equiv 0$ on $\widetilde{\Omega}$. Thus Theorem 1 is proved.

In the $C^{2}$ case, the comparison (2.2) is not uniform with regard to the size of $R$. The restriction $R<\alpha\left(x_{0}\right)$ is very inconvenient; and we believe that it could be removed.

We prove Propositions 2 and 3 in $\S \S 3-5$ below.

\section{Preliminary lemmas}

We let $L$ be a parabolic operator on $\mathbf{R}^{2}$; and in any domain $U$, denote by $\omega_{U}$ the associated parabolic measure. The next three lemmas are proved in [4]; the restriction that $a(x, t)$ is $C^{2}$ there can easily be removed.

Lemma A. Let $U=\{x>0, t>0\}, b>0$ and $0<t<b^{2} / 12 \Lambda_{2}$. Then $\omega_{U}^{(b, t)}(\{x=0\})<e^{-b^{2} / 144 t \Lambda_{2}}$.

Lemma B. Let $U=\{x>0, t>0\}$ and $0<\varepsilon<1$. Then $\omega_{U}^{(x, t)}(\{x=0\})>$ $1-\varepsilon$, for $x>0$ and $t>x^{2}\left(3 \varepsilon^{2} \Lambda_{1}\right)^{-1}$.

Lemma C. Let $b_{1}>b_{2}>0, U=\left\{-b_{2}<x<b_{1}, t>0\right\}$ and $S=\partial U \cap$ $\left\{x=b_{1},\right\}$; then $\omega_{U^{\prime}}^{(0, t)}(S) \leq b_{2} b_{1}^{-1}$, for $t>0$.

Lemma D. Corresponding to the parabolic operator $L$ on $\mathbf{R}^{2}$, define

$$
\begin{aligned}
& F(x, t)= \begin{cases}t^{-\Lambda_{2} / 2 \Lambda_{1}} e^{-x^{2} / 4 \Lambda_{1} t}, & \text { for } t>0, \\
0, & \text { for } t \leq 0 ;\end{cases} \\
& G(x, t)= \begin{cases}t^{-\Lambda_{1} / 2 \Lambda_{2}} e^{-x^{2} / 4 \Lambda_{2} t}, & \text { for } t>0, \\
0, & \text { for } t \leq 0 ;\end{cases}
\end{aligned}
$$




$$
H(x, t)= \begin{cases}\left(\frac{x}{t^{3 / 2}}\right)^{\Lambda_{1} / \Lambda_{2}} e^{-x^{2} / 4 \Lambda_{2} t}, & \text { for } x>0 \text { and } t>0, \\ 0, & \text { for } x \leq 0 \text { or } t \leq 0 ;\end{cases}
$$

and for $-\infty<M<\infty$, define

$$
K_{M}(x, t)= \begin{cases}\left(\frac{t+M x}{t^{3 / 2}}\right)^{\Lambda_{1} / \Lambda_{2}} e^{-x^{2} / 4 \Lambda_{2} t}, & \text { for } t>0 \text { and } t>-M x, \\ 0, & \text { for } t \leq 0 \text { or } t \leq-M x .\end{cases}
$$

Then $L F \geq 0$ on $\mathbf{R}^{2} \backslash\{(0,0)\}, L G \leq 0$ on $\mathbf{R}^{2} \backslash\{(0,0)\}, L H \leq 0$ on $\{x>0\}$, and $L K_{M} \leq 0$ on $\{t>-M x\}$.

Proofs contain straightforward differentiations and tedious estimations using the quadratic formula.

\section{4. $C^{2}$ Domains $\{t>\tau(x)\}$}

Let us retain all the assumptions from the second paragraph of $\S 2$, and assume, for convenience, that $\tau(0)=0$.

To prove Proposition 2, we need the following lemmas.

Lemma 1. Suppose that $\tau^{\prime}(0)=0$. Let $0<R<1$ and $(x, t) \in \Omega \cap\{|x| \leq R / 2$ and $\left.t \leq\left(A+1 / \Lambda_{1}\right) R^{2}\right\}$. Then

$$
\omega^{(x, t)}(\Phi(0, R))>C .
$$

Proof. Let $D=\left\{(y, s):|y|<R, s>-A R^{2} / 2\right\}$ and $S=\partial D \cap\left\{s=-A R^{2} / 2\right\}$. It follows from Lemma $A$ and the maximum principle that $\omega_{D}^{(y, s)}(S)>\frac{1}{2}$, when $|y|<R / 2$ and $s<-A R^{2} / 2+R^{2} / 1000 \Lambda_{2}$. Hence $\omega_{D}^{(x, t)}(S)>C>0$ by the Harnack principle. Since $\tau(x)>-A R^{2} / 2$ for $|x|<R,(4.1)$ follows from the maximum principle.

Lemma 2. Suppose that $\tau^{\prime}(0)=0$. Given $0<\varepsilon<1,0<\rho<1,|x|<\rho$ and $t>\rho^{2}\left(A / 2+1 / \varepsilon^{2} \Lambda_{1}\right)$, then $\omega_{\Omega \cap\{|x|<\rho\}}^{(x, t)}(\Phi(0, \rho))<\varepsilon$.

Proof. Let $D=\left\{|x|<\rho\right.$ and $\left.t>\rho^{2} A / 2\right\}$. We deduce from the maximum principle and Lemma $B$ that

$$
\omega_{\Omega \cap\{|x|<\rho\}}^{(x, t)}(\Phi(0, \rho))<\omega_{D}^{(x, t)}\left(\left\{|x|<\rho, t=\rho^{2} A / 2\right\}\right)<\varepsilon .
$$

Lemma 3. Suppose that $\tau^{\prime}(0)=0$. Given $0<\varepsilon, \rho<1$ and $0<r<\rho \varepsilon^{\Lambda_{2} / \Lambda_{1}}$, then $\omega(\Phi(0, r))<C \varepsilon$ at points $(\rho, t)$ and $(-\rho, t) \in \Omega$.

Proof. Let $u(x, t)=G\left(x, t+A r^{2}\right)$, where $G$ is the function defined in (3.2). We note that on $\Phi(0, r), u \geq C r^{-\Lambda_{1} / \Lambda_{2}}$; and that $u(\rho, t)$ attains its maximum value $\left(e \rho^{2} / 2 \Lambda_{1}\right)^{-\Lambda_{1} / 2 \Lambda_{2}}$ at $t=-A r^{2}+\rho^{2} / 2 \Lambda_{1}$. Because $u \geq 0$ and $L u \leq 0$ in $\Omega$, applying the maximum principle on $\Omega$, we obtain

$$
\omega^{(\rho, t)}(\Phi(0, r)) \leq C r^{\Lambda_{1} / \Lambda_{2}} u(\rho, t) \leq C\left(\frac{r}{\rho}\right)^{\Lambda_{1} / \Lambda_{2}}<C \varepsilon .
$$


Similarly, $\omega^{(-\rho, t)}(\Phi(0, r))<C \varepsilon$.

Next, we assume that $\tau^{\prime}(0)$ is negative, let

$$
m \equiv-\tau^{\prime}(0)>0 \cdot \text { and } \quad 0<r, \rho, R<\min \left\{1, m / 400 A^{2}\left(\Lambda_{1}+1\right)\right\} .
$$

It is clear that, for $|x|<m / 2 A$,

$$
\tau^{\prime}(x) \leq-\frac{m}{2} \text { and }|\tau(x)+m x|<m|x| / 4 .
$$

Lemma 4. Assume (4.2), then $\omega^{(x, 0)}(\Phi(0, R))>C$, where

$$
0<x<\sqrt{\Lambda_{1} m R} / 2 .
$$

Proof. Let $D=\left\{(x, t): 0<x<10 \sqrt{\Lambda_{1} m R}, t>-3 m R / 8\right\}$, and $S=\{(0, t)$ :

$-3 m R / 8<t<0\}$. We note from (4.2) that $R<m / 2 A$ and $10 \sqrt{\Lambda_{1} m R}<$ $m / 2 A$; thus it follows from (4.3) that $\tau(R / 2)<-3 m R / 8$ and that $\tau^{\prime}(x)<0$ for $0<x<10 \sqrt{\Lambda_{1} m R}$. From the maximum principle, we see that for $0<$ $x<\sqrt{\Lambda_{1} m R} / 2$,

$$
\begin{aligned}
\omega^{(x, 0)}\left(\Phi\left(0, \frac{R}{2}\right)\right) & >\omega_{D}^{(x, 0)}(S) \\
& >\omega_{\{x>0, l>-3 m R / 8\}}^{(x, 0)}(S)-\omega_{D}^{(x, 0)} \quad\left(\left\{x=10 \sqrt{\Lambda_{1} m R}\right\}\right) \\
& >\frac{1}{3}-\frac{1}{19} .
\end{aligned}
$$

The estimates $1 / 3$ and $1 / 19$ follow from Lemmas $B$ and $C$ respectively.

Lemma 5. Assume (4.2) and let $0<\varepsilon<\frac{1}{4}$. Then $\omega^{(x, t)}(\Phi(0, \rho))<\varepsilon$, when $(x, t) \in \Omega$ with $x \geq 24 \sqrt{\Lambda_{2} \log \frac{1}{\varepsilon} m \rho}$ and $\tau(\rho)<t<\tau(-\rho)$.

Proof. Let $D=\{(x, t): x>\rho$ and $t>\tau(\rho)\}$ and $S=\{(\rho, t): \tau(\rho)<t<$ $\tau(-\rho)\}$. It follows from the maximum principle that $\omega^{(x, t)}(\Phi(0, \rho))<\omega_{D}^{(x, t)}(S)$ for those $(x, t)$ satisfying the conditions above. Since $\tau(-\rho)-\tau(\rho)<(5 / 2) m \rho$ and $\rho<m$, we conclude the lemma by applying Lemma A to $\omega_{D}(S)$.

Lemma 6. Assume (4.2) and let $0<\varepsilon<10^{-5}$. Suppose that $r<10^{4} \rho \varepsilon^{\Lambda_{2} / \Lambda_{1}}$, then $\omega^{(x, \tau(-\rho))}(\Phi(0, r))<C \varepsilon$ for any $(x, \tau(-\rho)) \in \Omega \cap\{x>-\rho\}$.

Proof. Let $M=m / 2$ in (3.4) and

$$
u(x, t)=K_{m / 2}(x+\sqrt{m r}, t+2 m r) .
$$

Then $u \geq 0$ and $L u \leq 0$ in $\{t>-2 m r$ and $t+2 m r>-(m / 2)(x+\sqrt{m r})\}$; in particular, in $\Omega \cap\{\tau(r)<t<\tau(-\rho)$ and $x>-\rho\}$ because $\tau(x)>$ $\min \{(-5 / 4) m x,(-3 / 4) m x\}>-m x / 2-m^{3 / 2} r / 2-2 m r$ on $-\rho<x<r$. Because $r<m / 400$, it is easy to see that $u \geq C r^{-\Lambda_{1} / \Lambda_{2}}$ on $\Phi(0, r)$. Applying the maximum principle on $\Omega \cap\{x>-\rho$ and $\tau(r)<t<\tau(-\rho)\}$, we obtain

$$
\omega^{(x, \tau(-\rho))}(\Phi(0, r)) \leq C r^{\Lambda_{1} / \Lambda_{2}} u(x, \tau(-\rho)) .
$$


Let $\delta=\tau(-\rho)+2 m r, X=x+\sqrt{m r}$ and note that $m \rho / 4<\delta<4 m \rho$. Then we have, for $x>-\rho$,

$$
\begin{aligned}
u(x, \tau(-\rho)) & =\left(\frac{\delta+\frac{m}{2} X}{\delta^{3 / 2}}\right)^{\Lambda_{1} / \Lambda_{2}} e^{-X^{2} / 4 \Lambda_{2} \delta} \\
& \leq C\left[\left(\frac{1}{\delta}\right)^{\Lambda_{1} / 2 \Lambda_{2}}+\left(\frac{m}{\delta}\right)^{\Lambda_{1} / \Lambda_{2}}\left(\frac{|X|}{\delta^{1 / 2}}\right)^{\Lambda_{1} / \Lambda_{2}} e^{-X^{2} / 4 \Lambda_{2} \delta}\right] \\
& \leq C\left[\left(\frac{1}{\delta}\right)^{\Lambda_{1} / 2 \Lambda_{2}}+\left(\frac{m}{\delta}\right)^{\Lambda_{1} / \Lambda_{2}}\right] \\
& \leq C\left[\left(\frac{1}{m \rho}\right)^{\Lambda_{1} / 2 \Lambda_{2}}+\left(\frac{1}{\rho}\right)^{\Lambda_{1} / \Lambda_{2}}\right] \leq C\left(\frac{1}{\rho}\right)^{\Lambda_{1} / \Lambda_{2}} .
\end{aligned}
$$

If $r / \rho<10^{4} \varepsilon^{\Lambda_{2} / \Lambda_{1}}$, then

$$
\omega^{(x, \tau(-\rho))}(\Phi(0, r)) \leq C\left(\frac{r}{\rho}\right)^{\Lambda_{1} / \Lambda_{2}}<c \varepsilon .
$$

Proof of Proposition 2. Because (2.1) is a local property, we assume, as we may, that $x_{0}=0$ and recall that $\tau(0)=0$. Let $\varepsilon, r$, and $R$ satisfy the conditions in Proposition 2.

First, we assume that $\tau^{\prime}(0)=0$. Let $\ell_{1}=\{x=\varepsilon R, \tau(\varepsilon R)<t<(A+$ $\left.\left.1 / \Lambda_{1}\right) R^{2}\right\}, \ell_{2}=\left\{x=-\varepsilon R, \tau(-\varepsilon R)<t<\left(A+1 / \Lambda_{1}\right) R^{2}\right\}$ and $\ell_{3}=\{-\varepsilon R \leq$ $\left.x \leq \varepsilon R, t=\left(A+1 / \Lambda_{1}\right) R^{2}\right\}$. It is easy to verify that $\Omega \cap \partial \Gamma=\ell_{1} \cup \ell_{2} \cup \ell_{3}$. Because of the maximum principle, it suffices to verify (2.2) on $\ell_{1} \cup \ell_{2} \cup \ell_{3}$. We deduce from Lemma 1 that

$$
\omega^{(x, t)}(\Phi(0, R))>C \quad \text { on } \ell_{1} \cup \ell_{2} \cup \ell_{3} .
$$

Letting $\rho=\varepsilon R$, we deduce from (2.1) and Lemma 3 that

$$
\omega^{(x, t)}(\Phi(0, r))<\varepsilon \quad \text { on } \ell_{1} \cup \ell_{2} .
$$

Finally, for $(x, t) \in \ell_{3}$, it follows from the Markov property, the maximum principle and Lemmas 2 and 3 that

$$
\begin{aligned}
\omega^{(x, t)} & (\boldsymbol{\Phi}(0, r)) \\
& =\omega_{\Gamma}^{(x, t)}(\boldsymbol{\Phi}(0, r))+\int_{\ell_{1} \cup \ell_{2}} \omega^{(y, s)}(\Phi(0, r)) d \omega_{\Gamma}^{(x, t)}(y, s) \\
& \leq \varepsilon+\varepsilon=2 \varepsilon .
\end{aligned}
$$

Thus (2.2) holds, when $\tau^{\prime}(0)=0$.

Next, we assume that $\tau^{\prime}(0)=-m<0$. (The remaining case $\tau^{\prime}(0)>0$ can be treated similarly.) Let $\rho=\Lambda_{1} R / 10^{4} \Lambda_{2} \log 1 / \varepsilon, s_{1}=\left\{x=\sqrt{\Lambda_{1} m R} / 4, \tau(r)<\right.$ $t \leq \tau(-\rho)\}$ and $s_{2}=\left\{t=\tau(-\rho),-\rho<x \leq \sqrt{\Lambda_{1} m R} / 4\right\}$. It is clear that $\partial \Gamma \cap \Omega \cap\{t>\tau(r)\}=s_{1} \cup s_{2}$. By the maximum principle and the fact that $\omega^{(x, t)}(\Phi(0, r))=0$ for $t \leq \tau(r)$, we need only to prove (2.2) on $s_{1} \cup S_{2}$. The 
$x$-distance from any point on $s_{1} \cup s_{2}$ to $\partial \Omega$ is at most $\rho+\sqrt{\Lambda_{1} m R} / 4<$ $\sqrt{\Lambda_{1} m R} / 2$. We note from Lemma 4 that

$$
\omega^{(x, t)}(\Phi(0, R))>C \quad \text { on } s_{1} \cup s_{2} .
$$

Because $r<\left(\Lambda_{1} / \Lambda_{2}\right) R \varepsilon^{2+\Lambda_{2} / \Lambda_{1}}<10^{4} \rho \varepsilon^{\Lambda_{2} / \Lambda_{1}}$, we obtain

$$
\omega^{(x, \tau(-\rho))}(\Phi(0, r))<C \varepsilon
$$

on $s_{2}$, from Lemma 6. Since $\sqrt{\Lambda_{1} m R} / 4>24 \sqrt{\Lambda_{2} \log \frac{1}{\varepsilon} m \rho}$, (4.4) holds also on $s_{1}$. The conclusion (2.2) thus follows. And this completes the proof of Proposition 2.

The proof above is very lengthy, because we are unable to combine the case $\tau^{\prime}(0) \neq 0$, with the case $\tau^{\prime}(0)=0$. When $\tau^{\prime}(0) \neq 0$, implicitly we regard $x$ as a function of $t$ on $\partial \Omega$ near $(0,0)$.

\section{Lip $\frac{1}{2}$ DOMAINS $\{x>\chi(t)\}$}

We retain the assumptions from the third paragraph of $\S 2$, and assume that $\chi(0)=0$. The structure of the proof of Proposition 3 is the same as that of Proposition 2.

Lemma 7. Assume that $0<R<1$ and that $0<x<2 B \sqrt{R}$. Then

$$
\widetilde{\omega}^{(x, 0)}(\Psi(0, R))>c \text {. }
$$

Proof. Let $D=\{x>-B \sqrt{R}, t>-R\}$ and $S=\{x=-B \sqrt{R},-R<t<0\}$. From (2.3), the maximum principle and the Harnack principle, it follows that

$$
\widetilde{\omega}^{(x, 0)}(\Phi(0, R))>\omega_{D}^{(x, 0)}(S) \geq c \omega_{D}^{Q}(S),
$$

where $Q=\left(-B \sqrt{R}+\sqrt{\Lambda_{1} R} / 2,-R / 2\right)$. From Lemma $\mathrm{B}$, we deduce that $\omega_{D}^{Q}(S)>\frac{1}{2}$. Therefore (5.1) holds.

Lemma 8. Given $0<\varepsilon<\frac{1}{4}, 0<\delta<1$ and $-\delta<t<\delta$, then

$$
\widetilde{\omega}^{(x, t)}(\Psi(0, \delta))<\varepsilon
$$

if $x>\left(20 \sqrt{\Lambda_{2} \log 1 / \varepsilon}+B\right) \sqrt{\delta}$.

Proof. Let $D=\{x>B \sqrt{\delta}, t>-\delta\}$ and $S=\{x=B \sqrt{\delta},-\delta<t<\delta\}$. It follows from (2.3), Lemma A and the maximum principle that $\widetilde{\omega}^{(x, t)}(\Psi(0, \delta))<$ $\omega_{D}^{(. x, t)}(S)<\varepsilon$.

Lemma 9. Suppose that $0<\varepsilon<1,0<\delta<1$ and that $r<\delta \varepsilon^{2 \Lambda_{2} / \Lambda_{1}}$; then $\widetilde{\omega}^{(x, \delta)}(\Psi(0, r))<c \varepsilon$ for any $(x, \delta) \in \widetilde{\Omega}$.

Proof. Let $u(x, t)=G(x, t+2 r)$, with $G$ defined in (3.2). Because of (2.3), we have $u(x, t) \geq c r^{-\Lambda_{1} / 2 \Lambda_{2}}$ on $\Psi(0, r)$. Applying the maximum principle on 
$\widetilde{\Omega} \cap\{t>-r\}$, we obtain that for $(x, \delta) \in \widetilde{\Omega}$,

$$
\widetilde{\omega}^{(x, \delta)}(\Psi(0, r)) \leq c r^{\Lambda_{1} / 2 \Lambda_{2}} u(x, \delta) \leq c r^{\Lambda_{1} / 2 \Lambda_{2}}(\delta+2 r)^{-\Lambda_{1} / 2 \Lambda_{2}}<c \varepsilon .
$$

Proof of Proposition 3. We assume, as we may, that $t_{0}=0$ and $\chi(0)=0$. Let $\varepsilon, r$, and $R$ be as given and let $\delta=B^{2} R / 4000\left(\Lambda_{2} \log 1 / \varepsilon+B^{2}\right), \ell_{1}=$ $\{t=\delta, \chi(\delta)<x<B \sqrt{R} / 2\}$ and $\ell_{2}=\{x=B \sqrt{R} / 2,-r<t \leq \delta\}$. Clearly $\partial \Gamma \cap \widetilde{\Omega} \cap\{t>-r\}=\ell_{1} \cup \ell_{2}$. Again, because of the maximum principle it is enough to show (2.5) on $\ell_{1} \cup \ell_{2}$. Because the $x$-distance from any point on $\ell_{1} \cup \ell_{2}$ to $\partial \widetilde{\Omega}$ is at most $B \sqrt{\delta}+B \sqrt{R} / 2<B \sqrt{R}$, we deduce from Lemma 7 that

$$
\widetilde{\omega}^{(x, t)}(\Psi(0, R))>c \quad \text { on } \ell_{1} \cup \ell_{2} .
$$

Because $r<R \varepsilon^{2+2 \Lambda_{2} / \Lambda_{1}}<\delta \varepsilon^{2 \Lambda_{2} / \Lambda_{1}}$, we deduce from Lemma 9 that on $\ell_{1}$,

$$
\widetilde{\omega}^{(x, \delta)}(\Psi(0, r))<c \varepsilon .
$$

Since $B \sqrt{R} / 2>\left(20 \sqrt{\Lambda_{2} \log 1 / \varepsilon}+B\right) \sqrt{\delta}$, we see from Lemma 8 that (5.2) holds also on $\ell_{2}$. This completes the proof of Proposition 3 .

\section{DiRICHLET REGULAR DOMAINS AND THE MAXIMUM PRINCIPLE}

We consider the Dirichlet problem with continuous, compactly supported boundary data.

Theorem 2. Let $L$ be a parabolic operator and $D$ be a domain in $\mathbf{R}^{2}$. A point $\left(x_{0}, t_{0}\right) \in \partial D$ is a regular boundary point for $L$ if

(i) $\mathbf{R}^{2} \backslash D$ contains a line segment $\left\{\left(x_{0}, s\right): t_{0}-\delta \leq s \leq t_{0}\right\}$, or (ii) $\mathbf{R}^{2} \backslash D$ contains a region $\left\{(x, t): t \leq t_{0}\right.$ and $\left.-d \leq x-x_{0} \leq-b \sqrt{-\left(t-t_{0}\right)}\right\}$ for some $b, d>0$.

In particular, domains $\{t>\tau(x)\}$ with $\tau$ continuous on $\mathbf{R}$, and domains $\{x>\chi(t)\}$ with $\chi \operatorname{Lip} \frac{1}{2}$ on $\mathbf{R}$, are Dirichlet regular for $L$.

Proof. For the heat equation, regularity is proved in [2, Theorem 5] and [7, pp . 389-393] for the cases (i) and (ii) respectively.

Assume, for simplicity, that $\left(x_{0}, t_{0}\right)=(0,0)$. In the case (i), we let $v(x, t)=$ $\int_{-\delta}^{0} F(x, t-s) d s$, where $F$ is defined in (3.1), and note that $v$ attains a strict absolute maximum at $(0,0)$. In view of Lemma $\mathrm{D}, L v \geq 0$ on $\mathbf{R}^{2} \backslash\{(0, s)$ : $-\delta \leq s \leq 0\}$. Therefore $v(0,0)-v(x, t)$ is a barrier at $(0,0)$. Thus $(0,0)$ is a regular boundary point for $L$ [8].

In the case (ii), we follow closely the ideas in [7]. First we show that $(0,0)$ is a regular boundary point of the domain $U \equiv\{(x, t):-1<t<0,-b<$ $x / \sqrt{-t}<b\}$ : let $\Lambda_{1}$ and $\Lambda_{2}$ be the constants in (0.2) associated with $L$, and let $\beta>0$; for $(x, t) \in U$ with $-\log (-t)>\left(2 \beta \Lambda_{2} / \Lambda_{1}\right) e^{b^{2} / 4 \Lambda_{2}}$ the function $w$ defined by

$$
w(x, t)=-(-\log (-t))^{-\beta-1} e^{-x^{2} / 4 \Lambda_{2} t}+\frac{\Lambda_{1}}{2 \beta \Lambda_{2}}(-\log (-t))^{-\beta}
$$


is a barrier at $(0,0)$ for the domain $U(w>0, L w \leq 0$ and $w \rightarrow 0$ as $(x, t) \rightarrow(0,0)$ in $U)$. To deduce that $(0,0)$ is a regular boundary point for D, we follow step by step the proofs of [7, Theorems 2 and 3], where a similar statement for the heat equation is obtained. The proofs can be adapted to the parabolic operator $L$ in the obvious way, once the following property is proved.

Proposition 4. Given a parabolic operator $L$ and a domain $U \equiv\{(x, t):-1<$ $t<0,-b<x / \sqrt{-t}<b\}$, let $v$ be a bounded function satisfying $L v \geq 0$ on $U$ and having boundary values 0 on $\{x=-b \sqrt{-t}, t<0\}$, and 1 on $\{x=$ $b \sqrt{-t}, t<0\}$. Then $\lim \sup _{t \rightarrow 0} v(0, t)<1$.

Proof. Let

$$
\phi(\rho)=\int_{0}^{\rho} e^{s^{2} / 4} d s
$$

and define for $t<0$,

$$
u(x, t)= \begin{cases}\phi\left(\frac{x}{\sqrt{-\Lambda_{2} t}}\right), & x \geq 0, \\ \sqrt{\frac{\Lambda_{1}}{\Lambda_{2}} \phi}\left(\frac{x}{\sqrt{-\Lambda_{1} t}}\right), & x<0 .\end{cases}
$$

Straightforward differentiation shows that $L u \leq 0$. Let, for $t<0$,

$$
f(x, t)=\frac{u(x, t)-\sqrt{\frac{\Lambda_{1}}{\Lambda_{2}}} \phi\left(\frac{-b}{\sqrt{\Lambda_{1}}}\right)}{\phi\left(\frac{b}{\sqrt{\Lambda_{2}}}\right)-\sqrt{\frac{\Lambda_{1}}{\Lambda_{2}}} \phi\left(\frac{-b}{\sqrt{\Lambda_{1}}}\right)} .
$$

It is clear that $L f \leq 0, f=0$ on $x=-b \sqrt{-t}$ and $f=1$ on $x=b \sqrt{-t}$, and $0<f<1$ in $-b<x / \sqrt{-t}<b$. Moreover, $f$ is a constant on the line $\{x=0, t<0\}$. It follows from the maximum principle for bounded domains and the fact that $(0,0)$ is regular for $U$, that $\limsup _{t \rightarrow 0} v(0, t) \leq f(0,-1)<1$.

Theorem 3. Let $L$ be a parabolic operator and $u$ be a subsolution on $D=$ $\{t>\tau(x)\}$ with $\tau$ continuous on $\mathbf{R}$. Suppose that $u \leq 1$ on $D$ and that $\limsup u \leq 0$ as $(x, t)$ approaches any point on $\partial D$. Then $u \leq 0$ in $D$.

Proof. Fix $\left(x_{0}, t_{0}\right) \in D$. Let $\left(y, \tau\left(x_{0}\right)\right) \in D, M>5\left|y-x_{0}\right|$, and $D_{M}=$ $D \cap\left\{\left|x-x_{0}\right|<M\right\}$. Note that for sufficiently large $T>0, D_{M} \subseteq V \equiv\left\{\left|x-x_{0}\right|<\right.$ $\left.M, t-t_{0}>-T\right\} \backslash\left\{x=x_{0}, t \leq \tau\left(x_{0}\right)\right\}$, and that

$$
u\left(y, \tau\left(x_{0}\right)\right) \leq \omega_{v}^{\left.\left(y, \tau\left(y, \tau\left(x_{0}\right)\right)\right)\left(x_{0}\right)\right)}\left(\left\{\left|x-x_{0}\right|=M\right\} \cap \partial V\right)
$$

by the maximum principle for bounded domains. The right hand side is bounded above by $\left|y-x_{0}\right|\left(M-\left|y-x_{0}\right|\right)^{-1}$ in view of Lemma C. Letting $M \rightarrow \infty$, we have $u\left(y, \tau\left(x_{0}\right)\right) \leq 0$. Thus $u \leq 0$ on $D \cap\left\{t=\tau\left(x_{0}\right)\right\}$. We deduce from Lemma $\mathrm{A}$ and the maximum principle for bounded domains that $u\left(x_{0}, t_{0}\right) \leq 0$.

Theorem 4. Let $L$ be a parabolic operator and $u$ be a subsolution on $D=\{x>$ $\chi(t)\}$ with $\chi$ Lip $\frac{1}{2}$ on $\mathbf{R}$. Suppose that $u \leq 1$ on $D$ and that $\lim \sup u \leq 0$ at every boundary point of $D$. Then $u \leq 0$ in $D$. 
Proof. Choose $B>1$, so that

$$
|\chi(t)-\chi(s)| \leq B|t-s|^{1 / 2} \quad \text { for all } t, s \in R .
$$

Given $0<\varepsilon<\frac{1}{2}$ and $\left(x_{0}, t_{0}\right) \in D$, we need to show that $u\left(x_{0}, t_{0}\right)<\varepsilon$. The fact that every boundary point of $D$ is Dirichlet regular is implicitly used in the proof.

Choose and fix a point $Q$ on $\partial \mathrm{D}$ with $t$-coordinate $>t_{0}$, so that after a translation, $Q=(0,0), t_{0}<0$ and $\left|x_{0}\right|<\beta \sqrt{-t_{0}}$.

Choose and fix a number $k>2 / \varepsilon$ which satisfies

$$
\left[\phi\left(\frac{B}{\sqrt{\Lambda_{2}}}\right)-\sqrt{\frac{\Lambda_{1}}{\Lambda_{2}}} \phi\left(\frac{-B}{\sqrt{\Lambda_{1}}}\right)\right]\left[\phi\left(\frac{k B}{\sqrt{\Lambda_{2}}}\right)-\sqrt{\frac{\Lambda_{1}}{\Lambda_{2}}} \phi\left(\frac{-B}{\sqrt{\Lambda_{1}}}\right)\right]^{-1}<\varepsilon,
$$

where $\phi$ is the function in (6.2). Let

$$
U=\{t<0,-B \sqrt{-t}<x<k B \sqrt{-t}\},
$$

and define for $t<0$,

$$
v(x, t)=\frac{u(x, t)-\sqrt{\frac{\Lambda_{1}}{\Lambda_{2}}} \phi\left(\frac{-B}{\sqrt{\Lambda_{1}}}\right)}{\phi\left(\frac{k B}{\sqrt{\Lambda_{2}}}\right)-\sqrt{\frac{\Lambda_{1}}{\Lambda_{2}}} \phi\left(\frac{-B}{\sqrt{\Lambda_{1}}}\right)},
$$

where $u$ is the function in (6.3). Clearly $L v \leq 0, v=1$ on $S \equiv\{x=$ $k B \sqrt{-t}, t<0\}$, and $v=0$ on $\{x=-B \sqrt{-t}, t<0\}$; moreover, we see from (6.4) that $\sup _{-B<x / \sqrt{-t}<B} v(x, t)<\varepsilon$, in particular $v\left(x_{0}, t_{0}\right)<\varepsilon$.

Let $T>-10 t_{0}$ and $U_{T}=U \cap\{t>-T\}$. It follows from the maximum principle for bounded domains that

$$
u\left(x_{0}, t_{0}\right)<v\left(x_{0}, t_{0}\right)+\omega_{U_{T}}^{\left(x_{0}, t_{0}\right)}\left(\partial U_{T} \cap\{t=-T\}\right) .
$$

After a change of scales, we obtain for sufficiently large $T$,

$$
\omega_{U_{T}}^{\left(x_{0}, t_{0}\right)}\left(\partial U_{T} \cap\{t=-T\}\right) \leq w\left(\frac{x_{0}}{\sqrt{T}}, \frac{t_{0}}{T}\right),
$$

where $w$ is the function defined in (6.1). Letting $t \rightarrow \infty$, we conclude that $u\left(x_{0}, t_{0}\right)<\varepsilon$. This concludes the proof.

Standard deductions give the maximum principle for bounded solutions of $L u=0$, and the uniqueness theorem for the Dirichlet problem in domains described in Theorems 3 and 4.

\section{REFERENCES}

1. E. Fabes and C. Kenig, Examples of singular parabolic measures and singular transition probability density, Duke Math. J. 48 (1981), 845-856.

2. R. Kaufman and J.-M. Wu, Parabolic potential theory, J. Diff. Eqs. 43 (1982), 204-234. 
3. __ Parabolic measure on domains of class Lip $\frac{1}{2}$, Compositio Math. 65 (1988), 201-207.

4. __ An example of highly singular parabolic measure, Ann. Probability 16 (1988), 18211831.

5. __ Dirichlet problem of heat equation for $C^{2}$ domains, J. Diff. Eqs., (to appear).

6. J. L. Lewis and J. Silver, Parabolic measure and the Dirichlet problem for the heat equation in two dimensions, preprint.

7. I. G. Petrowsky, Zur ersten Randwertaufgaben der Warmeleitungsgleichung, Compositio Math. 1 (1935), 383-419.

8. N. A. Watson, Green functions, potentials and the Dirichlet problem for the heat equation, Proc. London Math. Soc. 33 (3) (1976), 251-298.

UNIVERSITY OF IllinoIs, Urbana, ILlinOIS 61801 